\title{
A comparative study of next-generation sequencing and fragment analysis for the detection and allelic ratio determination of FLT3 internal tandem duplication
}

\author{
Jin Ju Kim, Kwang Seob Lee ${ }^{1}$, Taek Gyu Lee², Seungjae Lee ${ }^{1}$, Saeam Shin ${ }^{1 *}$ (1) and Seung-Tae Lee ${ }^{1}$
}

\begin{abstract}
Background: Currently, FLT3 internal tandem duplication (ITD) is tested by fragment analysis. With next-generation sequencing (NGS), however, not only FLT3 ITD but also other mutations can be detected, which can provide more genetic information on disease.

Methods: We retrospectively reviewed the results of two tests-fragment analysis and a custom-designed, hybridization capture-based, targeted NGS panel_-performed simultaneously. We used the Pindel algorithm to detect FLT3 ITD mutations.

Results: Among 277 bone marrow aspirate samples tested by NGS and fragment analysis, the results revealed 99.6\% concordance in FLT3 ITD detection. Overall, the allele frequency (AF) attained by NGS positively correlated with the standard allelic ratio (AR) attained by fragment analysis, with a Spearman correlation coefficient $(r)$ of 0.757 (95\% confidence interval: $0.627-0.846 ; p<0.001$ ). It was concluded that an AF of 0.11 attained by NGS is the most appropriate cutoff value (with $85.3 \%$ sensitivity and $86.7 \%$ specificity) for high mutation burden criterion presented by guidelines.

Conclusion: Sensitive FLT3 ITD detection with comprehensive information of other mutation offered by NGS could be a useful tool in clinical laboratories. Future studies will be needed to evaluate and standardize NGS AF cutoff to predict actual clinical outcomes.
\end{abstract}

Keywords: Acute myeloid leukemia, FLT3 internal tandem duplication, Fragment analysis, Allele ratio

\section{Introduction}

Internal tandem duplication (ITD) in the Fms-related receptor tyrosine kinase 3 (FLT3) gene is found in approximately 20 to $30 \%$ of cases of normal karyotype acute myeloid leukemia (AML) [1]. FLT3 ITD is a driver of leukemogenesis, resulting in constitutive activation of

\footnotetext{
* Correspondence: saeam0304@yuhs.ac

'Department of Laboratory Medicine, Yonsei University College of Medicine, Severance Hospital, 50-1 Yonsei-ro, Seodaemun-gu, Seoul 03722, Republic of Korea

Full list of author information is available at the end of the article
}

the FLT3 receptor and autonomous proliferation of cells [2]. It is transcribed in-frame and occurs in from three to a few hundred base pairs commonly in the juxtamembrane domain region [3]. Patients with FLT3 ITD are associated with a poor prognosis due to a high risk of recurrence and shorter overall survival [1]. Recently, two FLT3 tyrosine kinase inhibitors, midostaurin and gilteritinib, were approved and are used in patients with FLT3-mutated AML $[4,5]$.

Polymerase chain reaction (PCR) with capillary electrophoresis-based DNA fragment analyses is the

C C The Author(s). 2022 Open Access This article is licensed under a Creative Commons Attribution 4.0 International License, which permits use, sharing, adaptation, distribution and reproduction in any medium or format, as long as you give appropriate credit to the original author(s) and the source, provide a link to the Creative Commons licence, and indicate if changes were made. The images or other third party material in this article are included in the article's Creative Commons licence, unless indicated otherwise in a credit line to the material. If material is not included in the article's Creative Commons licence and your intended use is not permitted by statutory regulation or exceeds the permitted use, you will need to obtain permission directly from the copyright holder. To view a copy of this licence, visit http://creativecommons.org/licenses/by/4.0/ The Creative Commons Public Domain Dedication waiver (http://creativecommons.org/publicdomain/zero/1.0/) applies to the data made available in this article, unless otherwise stated in a credit line to the data. 
current standard method for the detection and quantification of FLT3 ITD [6-8]. Clinical guidelines, such as the European LeukemiaNet (ELN) and National Comprehensive Cancer Network consensus (NCCN) guidelines, define a FLT3 ITD allelic ratio cutoff of 0.5 as measured by fragment analysis as the optimal riskstratification criterion in AML [6, 9].

In the last few years, many molecular diagnostic laboratories have come to employ next-generation sequencing (NGS), which has the advantage of identifying AML-related somatic mutations in multiple genes. Quantitative analysis is also possible using NGS; however, the detection and quantification of large insertion/ deletion (indel) variants are problematic.

In this work, we retrospectively analyzed the clinical data of patients who underwent FLT3 ITD tests using fragment analysis and NGS applied at the primary source to assess the correlation between these two methods of mutation detection and burden measurement.

\section{Materials and methods Patients and samples}

This study protocol was approved by the Institutional Review Board of Yonsei University Severance Hospital (approval no. 2021-0389-001). We retrospectively reviewed the concurrent test results of fragment analysis for FLT3 ITD and a targeted NGS panel deployed in our laboratory from January 2017 to March 2021. Patients' diagnosis and response criteria were followed with the 2016 World Health Organization classification of myeloid neoplasms and acute leukemia and 2017 ELN recommendations $[6,10]$. Samples were referred to the laboratory at diagnosis and follow-up for hematologic neoplasms. For both fragment analysis and NGS, genomic DNA was extracted from bone marrow or wholeblood samples using the QIAamp DNA Blood Mini Kit (Qiagen, Venlo, The Netherlands).

\section{Capillary electrophoretic fragment analysis}

PCR was performed on genomic DNA using an AccuPower HotStart PCR Premix (Bioneer, Daejeon, Korea) and specific primers [11] (forward: 5'-FAM- TGCCTA TTCCTAACTGACTCATCA--3', reverse:5' - TCTTTG TTGCTGTCCTTCCA $-3^{\prime}$ ) to amplify FLT3 exon 14 and 15 regions with following conditions: $94^{\circ} \mathrm{C}$ for 5 min followed by 35 cycles of $94{ }^{\circ} \mathrm{C}$ for $30 \mathrm{~s}, 60^{\circ} \mathrm{C}$ for $30 \mathrm{~s}$ and $72{ }^{\circ} \mathrm{C}$ for $60 \mathrm{~s}$, and final extension at $72{ }^{\circ} \mathrm{C}$ for $7 \mathrm{~min}$. Thereafter, $10 \mu \mathrm{L}$ of the mixture of $0.5 \mu \mathrm{L}$ fragmentlength standard (GeneScan-600 LIZ Size Standard, Thermo Fisher Scientific, Foster City CA, USA) and $10 \mu \mathrm{L}$ HiDi formamide (Thermo Fisher Scientific) was added to $1 \mu \mathrm{L}$ of 30 times diluted PCR product. After the initial denaturation of this mixture at $95^{\circ} \mathrm{C}$, size- separation by capillary electrophoresis was performed on a 3130 Genetic Analyzer (Thermo Fisher Scientific) using the separation matrix POP-7 polymer (Thermo Fisher Scientific). Data analysis was performed using the GeneMapper version 3.2 software program (Thermo Fisher Scientific). The FLT3 ITD allelic ratio (AR) is calculated as the ratio of the peak height of the mutant product to the peak height of the wild-type product. The detection limit of PCR-based capillary electrophoresis fragment analysis used in this study was AR of 0.01 . A manual review of the FLT3 ITD region was performed in all cases.

\section{Targeted NGS}

NGS was performed with custom probes targeting 497 genes related to hematologic neoplasms (Supplementary Table 1). The DNA probe was used, and the FLT3 ITD region (FLT3 exon 14, exon 15, and intron 14) was tiled with $2 \times$ density. Prepared libraries were then hybridized with probes and sequenced on the NextSeq 550Dx instrument (Illumina, San Diego, CA, USA). The BurrowsWheeler alignment tool was used for sequence alignment. The Pindel algorithm, which was implemented in the DxSeq Analyzer (Dxome, Seoul, Korea), was used to detect FLT3 ITD mutations. The bioinformatics pipelines used the analysis was presented in Supplementary Fig. 1. The FLT3 ITD allelic frequency (AF) was calculated from the number of sequencing reads showing internal tandem duplication versus the total sequence.

\section{Statistical analysis}

The statistical analysis was performed using MedCalc version 18.2.1 (MedCalc Software, Mariakerke, Belgium). Continuous variables are described using mean values, whereas categorical variables are presented as percentages. Concordance was measured using the percent of agreement between the two assays. A metric variable correlation between AR values obtained by fragment analysis versus AF values obtained by NGS was conducted using Passing Bablok regression analysis with Spearman correlation coefficient. A receiver-operating characteristic (ROC) analysis was performed to determine the sensitivity, specificity, and optimal diagnostic cutoff values of NGS in screening for high FLT3 ITD burden cases. The most appropriate cutoff value was chosen according to ROC analysis, and the area under the ROC curve (AUC) was calculated. Results were considered statistically significant at the level of $p<0.05$.

\section{Results}

Clinical and molecular characteristics of FLT3 ITD-positive cases identified by NGS

Among 277 samples tested NGS and fragment analysis, 260 cases were collected from initial samples of patients, 
including AML $(n=236)$, B-lymphoblastic leukemia $(n=$ $5)$, myelodysplastic syndrome/cytopenia $(n=13)$, mixedphenotype acute leukemia $(n=2)$, myeloid sarcoma $(n=$ $3)$ and pure erythroid leukemia $(n=1)$. The remaining 17 samples were follow-up samples taken from AML patients. Among 277 samples, FLT3 ITD was detected by both methods in 64 cases and only by NGS in one case. (Table 1). 61 initial samples with FLT3 ITD positive by both methods were collected from AML patients, including AML with mutated NPM1 $(n=24)$, AML with maturation $(n=14)$, AML with myelodysplasia-related change $(n=5)$, acute myelomonocytic leukemia $(n=5)$, AML with biallelic mutation of CEBPA $(n=3)$, acute promyelocytic leukemia $(n=3)$, AML with mutated RUNX1 $(n=2)$, AML with $\mathrm{t}(8 ; 21)(\mathrm{q} 22 ; \mathrm{q} 22.1)(n=2)$, acute monocytic leukemia $(n=2)$ and AML with inv. (16)(p13.1q22) $(n=1)$ (Supplementary Table 2). Aside from 3 acute promyelocytic leukemia patients and one patients without further treatment record, all patients received either standard $3+7$ induction chemotherapy $(n=35)$ or hypomethylating agent therapy $(n=22) .19$ out of 60 patients with treatment medical record are treated with FLT3 inhibitor.

Overall, there were 56 distinct types of FLT3 ITD identified, ranging in size from 18 up to $216 \mathrm{bp}$, with 48 (85.7\%), $3(5.4 \%)$, and $5(8.9 \%)$ of the 56 types falling in the ranges of less than 100,100 to 150 , and greater than $150 \mathrm{bp}$, respectively. In addition, 41 (73.2\%), 8 (14.3\%) and $7(12.5 \%)$ ITDs showed insertion sites at exon 14, exon 15 , and intron 14 , respectively. We observed simple internal tandem duplication, as well as insertion of random extra nucleotides in the 56 types of FLT3 ITDs: 29 (51.8\%) demonstrated simple tandem duplications with a size range of $18-216 \mathrm{bp}$; and other 27 (48.2\%) had insertion of extra nucleotides of unknown origin with a size range of 24-167 bp (Supplementary Table 2).

There were 24 samples from 8 patients assessed by both methods. The studied patients' clinical and biological characteristics are shown in Supplementary Table 3. Four patients with FLT3 ITD detected by NGS failed to respond to the chemotherapy.

\section{Comparison of FLT3 ITD detection by NGS and fragment analysis}

NGS demonstrated $99.6 \%$ concordance (kappa value: 0.990, 95\% confidence interval (CI): 0.970-1.000) with fragment analysis in FLT3 ITD detection, with 65 NGSpositive (size range: $18-216 \mathrm{bp}$ ) and 212 NGS-negative cases consistently ruled as positive and negative by fragment analysis, respectively. The AR of FLT3 ITD was estimated to be 0.02 to 28.34 per PCR fragment analysis and the AF was estimated to be 0.005 to 0.937 per NGS analysis. In the single discordant case, FLT3 ITD mutation was not detected by fragment analysis but exhibited a low mutation burden by NGS (AF of 0.006, 0.6\%).

Overall, the AF attained by NGS positively correlated with the AR attained by fragment analysis, with a spearman rank correlation coefficient $(r)$ of 0.757 (95\% CI: 0.627-0.846; $p<0.001$ ) (Fig. 1). In comparing the tumor burden detected by the two methods, different cutoffs were applied due to the difference in burden calculation formula. A value of 0.5 as the cutoff to discern between low and high mutation burdens for fragment analysis was used according to the 2017 ELN guidelines, and a value of 0.33 for NGS was used to concord with the cutoff value by fragment analysis. The NGS results

Table 1 Summary of diagnosis and FLT3 ITD results from patients

\begin{tabular}{|c|c|c|c|c|c|}
\hline \multirow[t]{2}{*}{ Diagnosis } & \multirow{2}{*}{$\begin{array}{l}\text { Sample, } \\
n\end{array}$} & \multicolumn{2}{|l|}{ NGS } & \multicolumn{2}{|l|}{ Fragment analysis } \\
\hline & & $\begin{array}{l}\text { FLT3 ITD-positive cases, } n \\
\text { (\%) }\end{array}$ & $\begin{array}{l}\text { Mean allelic frequency } \\
\text { (range) }\end{array}$ & $\begin{array}{l}\text { FLT3 ITD-positive case, } n \\
(\%)\end{array}$ & $\begin{array}{l}\text { Mean allelic ratio } \\
\text { (range) }\end{array}$ \\
\hline Initial & 260 & $61(23.5)$ & $0.16(0.01-0.94)$ & $61(23.5)$ & $1.24(0.02-28.34)$ \\
\hline AML (APL included) & 236 & $61(25.8)$ & $0.16(0.01-0.94)$ & $61(25.8)$ & $1.24(0.02-28.34)$ \\
\hline BLL & 5 & 0 & - & 0 & - \\
\hline MDS/cytopenia & 13 & 0 & & 0 & \\
\hline MPAL & 2 & 0 & - & 0 & - \\
\hline Myeloid sarcoma & 3 & 0 & - & 0 & - \\
\hline Pure erythroid leukemia & 1 & 0 & - & 0 & - \\
\hline Follow-up & 17 & $4(23.5)$ & $0.26(0.01-0.78)$ & $3(17.6)$ & $4.77(0.62-12.56)$ \\
\hline $\begin{array}{l}\text { AML in complete } \\
\text { remission }\end{array}$ & 13 & 0 & - & 0 & - \\
\hline $\begin{array}{l}\text { AML in relapse/residual } \\
\text { blast }\end{array}$ & 4 & $4(100.0)$ & $0.26(0.01-0.78)$ & $3(75.0)$ & $4.77(0.62-12.56)$ \\
\hline Total & 277 & $65(23.5)$ & $0.15(0.01-0.94)$ & $64(23.1)$ & $1.39(0.02-28.34)$ \\
\hline
\end{tabular}

Abbreviations: AML, acute myeloid leukemia; APL, acute promyelocytic leukemia; BLL, B-lymphoblastic leukemia; MDS, myelodysplastic syndrome; MPAL, mixedphenotype acute leukemia; NGS, next-generation sequencing, ITD, internal tandem duplication 


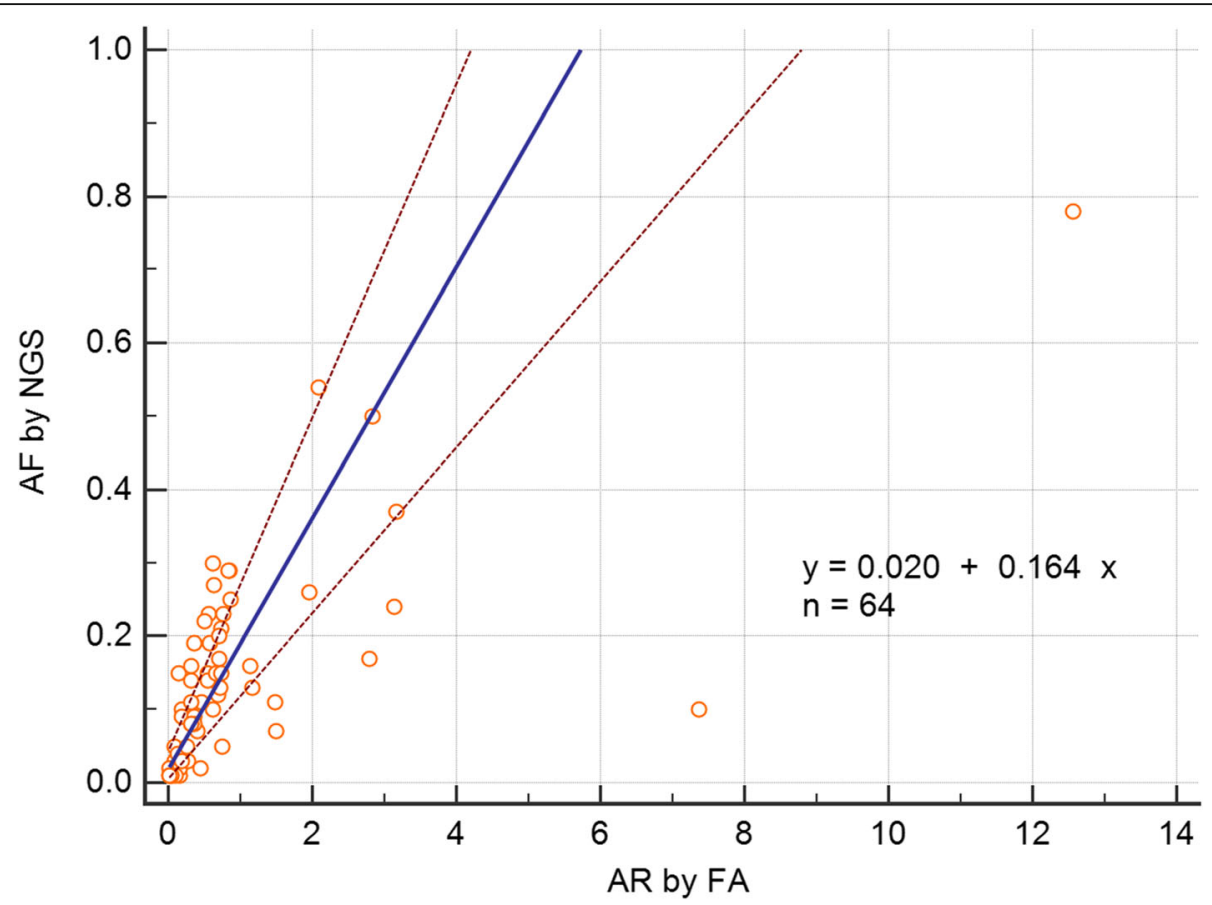

Fig. 1 Correlation of the FLT3 ITD mutant burden as assessed by the two study methods; Allelic ratio (AR) values from fragment analysis and allele frequency (AF) values from NGS are indicated, respectively. Abbreviations: AF, allelic frequency; NGS, next generation sequencing; AR, allelic ratio; FA, fragment analysis

demonstrated fair agreement (kappa value: 0.14) with fragment analysis in five of $34(14.7 \%)$ high-mutationburden cases and 30 of 30 (100.0\%) low-mutationburden cases. Overall agreement was $54.7 \%$ (35/ 64) (Table 2).

We performed a ROC curve analysis to specify the appropriate cutoff value for AF per the NGS test method to determine better the degree of tumor burden adopted by the ELN guideline. Based on the results of the ROC curve analysis, the cutoff value to achieve optimal sensitivity and specificity during NGS was 0.11 (Fig. 2). If an allelic ratio of 0.11 for NGS was chosen as a new cutoff value, the sensitivity and specificity values for diagnosis were $85.3 \%$ (95\% CI: $68.9-95.0 \%)$ and $86.7 \%$ (95\% CI: 69.3-96.2\%), respectively.

\section{Discussion}

FLT3 ITD mutation is a well-known predictive factor for poor prognosis in AML [12]. According to recommendations, the higher relapse rates and shorter overall survival times associated with FLT3 ITDs are influenced by mutation burden, at the boundary of 0.5 , such that a highly accurate measurement of AR is necessary [13]. Patients with FLT3 ITD mutations carry a particularly poor prognosis such that allogeneic bone marrow transplantations during the first period of remission in this population are usually performed $[14,15]$. However, relapse following transplant occurs frequently $[16,17]$.

Recently, FLT3 inhibitors were introduced, leading FLT3 ITD to become an even more important indicator for determining subsequent treatment modalities [18, 19]. Minimal residual disease (MRD) studies conducted using quantitative reverse-transcription PCR have made it clear that FLT3 ITD MRD levels following induction chemotherapy are a predictive marker of the duration of complete remission. However, sequence-specific PCR is burdensome to perform, difficult to standardize, and not routinely available outside of a research setting [20-23].

NGS enables the detection of subclinical disease in AML and allows the identification of the clonal

Table 2 Concordance of FLT3 ITD mutation burden classification between NGS and fragment analysis

\begin{tabular}{llll}
\hline Fragment analysis & Next-generation sequencing & Total & $\begin{array}{c}\% \\
\text { Agreement }\end{array}$ \\
\cline { 2 - 4 } & Low mutation burden & High mutation burden & $30(46.9 \%)$ \\
Low mutation burden & 30 & 0 & $34(53.1 \%)$ \\
High mutation burden & 29 & 5 & $\mathbf{5 4 . 7 \%}$ \\
Total & $\mathbf{5 9 ( \mathbf { 9 2 . 2 } \% )}$ & $\mathbf{5 ( 7 . 8 \% )}$ & $\mathbf{6 4}$ \\
\hline
\end{tabular}




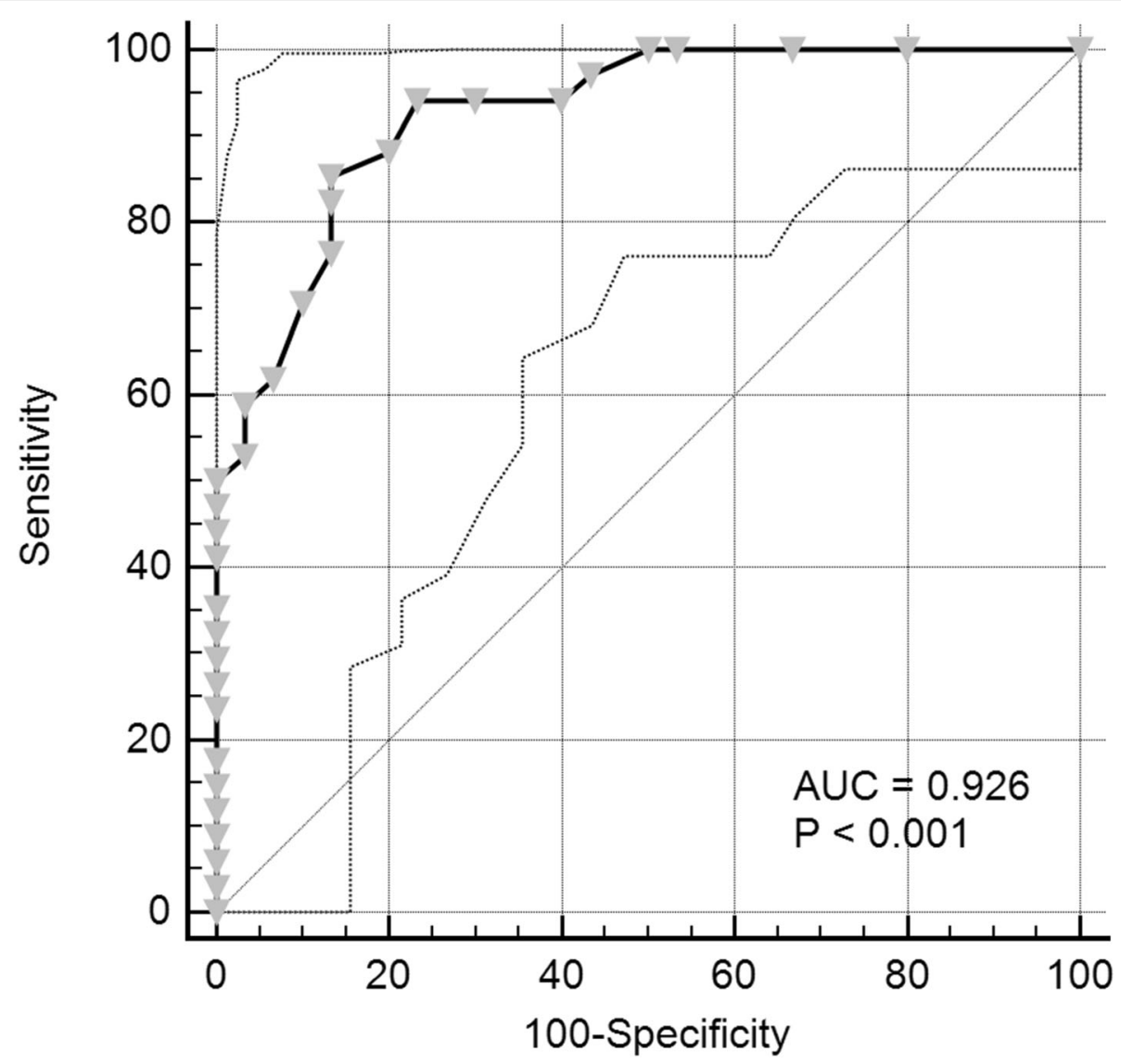

Fig. 2 ROC curves were calculated for revealing a high FLT3 ITD burden using AF as determined by NGS. A high FLT3 ITD burden was indicated by an AR value of greater than 0.5 by fragment analysis. By applying the cutoff value of 0.11 , the AUC was 0.926 (95\% Cl: 0.833-0.977). ROC curve (solid line) with $95 \%$ confidence interval (dashed lines). The dotted line is a diagonal line

composition and dominance of FLT3 ITD mutations. Patients may harbor multiple FLT3 ITD clones, and clonal allelic burden change can be monitored by NGS [20]. As the detection sensitivity of fragment analysis is known to be about $1 \sim 5 \%$ of the mutant allele burden due to PCR bias [24, 25], NGS is reported to demonstrate better analytical sensitivity than traditional fragment analysis [22, 26]. If the disease burden is lower than $1 \%$, it may not be detectable using the fragment method but could be detected by the NGS, and this low burden of disease could be linked to relapse after intensive chemotherapy or transplantation. Moreover, with the improved detection sensitivity of NGS relative to that of fragment analysis, the lack of a need for sequence-specific probes increases the flexibility of NGS deployment [20-23].

In this study, we used hybridization and capture enrichment with Illumina sequencing to sequence FLT3 and 496 other genes to determine the identification of FLT3 ITD in a multigene NGS panel. We used the Pindel algorithm for the variant caller system, which is a pattern-growth algorithm using read pairs wherein one is partially or completely unaligned and is reported to be reliable for FLT3 ITD mutation detection, thus identifying the position and length of FLT3 ITD [21, 27]. Our results showed that the NGS method using the Pindel algorithm exhibited $99.6 \%$ concordance with fragment analysis.

One AML case (Patient 7) with discordant results from NGS and fragment analysis, respectively, demonstrated a relatively low mutation burden $(0.6 \%)$ per NGS; this result was obtained from patient's follow-up sample. A small peak was detected in the fragment analysis from the follow-up sample, but the calculated AR was below the detection limit of fragment analysis that was considered negative. This patient harbored IDH2 (one missense), DNMT3A (one missense and one nonsense), and NCOR (one missense) gene mutations together with FLT3 ITD. After six cycles of hypomethylating therapy, the patient underwent NGS as well as FLT3 ITD fragment analysis. The mutation burden change of each mutation in this case is depicted in Fig. 3. Mutations other than FLT3 ITD revealed by fragment analysis persisted in follow-up samples, and bone marrow aspirate showed 


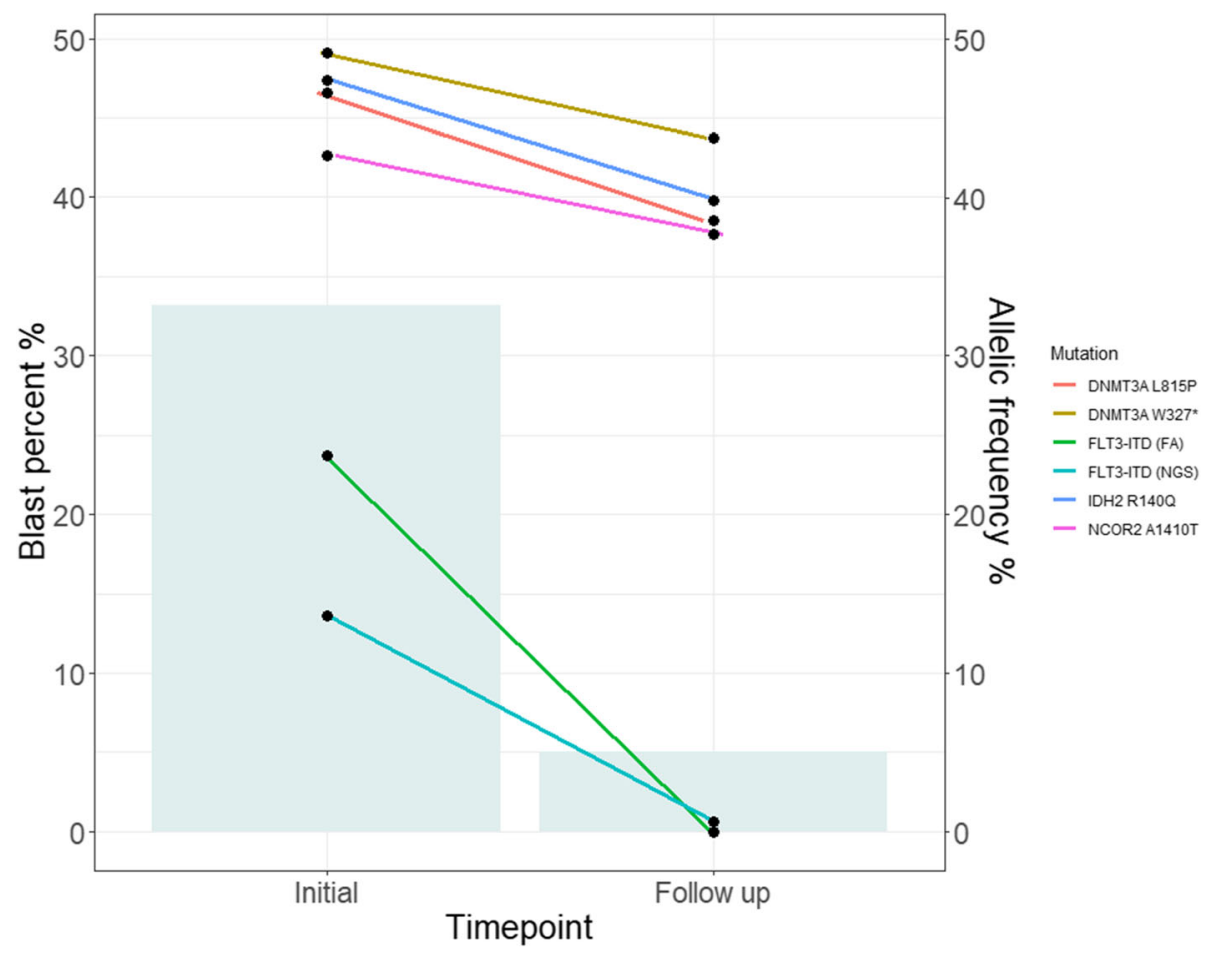

Fig. 3 Change in AF values of somatic mutations in an AML case with discordant results from NGS and fragment analysis, respectively (Patient 7). The FLT3 ITD AR was calculated by fragment analysis was converted into an AF value according to the following equation; $A F(\%)=A R /(1-$ $A R)^{*} 100$. The box represents the blast percentage observed in a bone marrow aspirate smear sample

a residual blast presence of approximately $5 \%$. This patient ultimately relapsed four months into the follow-up period; this outcome suggested that low-level ITD alleles were missed by PCR, yet FLT3 ITD could be real. NGS presents a better potential to be incorporated into the clinical field in ITD detection. Like in our case, samples with a low mutation burden near the detection limit of the conventional fragment analysis could be detected more by the NGS method.

Quantitative analysis revealed a moderate degree of correlation between two methods in clinical samples $(r=$ 0.757; slope: 0.164). The reason for this low regression slope is thought to be partly due to the well-known tendency of the Pindel algorithm to underestimate the mutant allele fraction [23, 26, 27]. Also, the NGS capture efficiency bias could be the another reason for underestimating the mutation burden in detecting large indels [23, 28]. Accordingly, the ROC curve was analyzed, and, through our results, a cutoff value of $0.11-$ which is lower than 0.33, a cutoff corresponding to an AR value of 0.5could be calculated. The diagnostic sensitivity in this case was $85.3 \%$, which was an improved result relative to that achieved when applying the existing criterion to determine the degree of mutation burden. Our result was suggested that the criterion of AF from NGS to determine the degree of mutation burden-which is judged to be related to the clinical prognosis in the current guideline-should be applied differently from 0.33 . Future studies should evaluate whether the AF cutoff using NGS has a clinical prognostic value. When using NGS, various factors such as probe design, sequencing chemistry, and bioinformatics pipeline affect the results, so standardization efforts on setting cutoff will also be needed.

Sensitive yet reliable detection of FLT3 ITD is required in the clinical field, and monitoring of disease status offers a significant advantage for clinicians. Applying NGS analysis could help guide decision-making, including transplantation and timely use of FLT3 inhibitors. Using the NGS method, FLT3 ITD can be identified sensitively through a single test and other gene mutation patterns, which can increase comprehensive understanding of the disease. In addition, according to the assay and bioinformatics algorithm used, the laboratory should establish a standard for high mutation burden criterion based on established guidelines.

\section{Supplementary Information}

The online version contains supplementary material available at https://doi. org/10.1186/s13000-022-01202-x.

Additional file 1 Supplementary Table 1. List of genes included in the hematologic malignancy-target gene panel. Supplementary 
Table 2. Clinical information and FLT3 ITD results of samples obtained from patients. Supplementary Table 3. Clinical and biological characteristics of the studied patients with follow up samples.

Supplementary Fig. 1. The bioinformatics pipelines used in this study Supplementary Fig. 2. FLT3 ITD detection result by fragment analysis and by NGS in acute myeloid leukemia patient with discrepant case (Patient 7).

\section{Authors' contributions}

Jin Ju Kim: Data curation, formal analysis, writing —original draft. Kwang Seob Lee: Data curation. Taek Gyu Lee: Data curation and writing draft. Seungjae Lee: Formal analysis. Saeam Shin: Conceptualization, supervision, writing - review and editing. Seung-Tae Lee: Resources. All authors read and approved the final manuscript.

\section{Funding}

Supported by a grant from the National Research Foundation of Korea (2021R111A1A01045980).

\section{Declaration}

\section{Competing interests}

The authors declare that they have no competing interests.

\section{Author details}

${ }^{1}$ Department of Laboratory Medicine, Yonsei University College of Medicine, Severance Hospital, 50-1 Yonsei-ro, Seodaemun-gu, Seoul 03722, Republic of Korea. ${ }^{2}$ Brain Korea 21 PLUS Project for Medical Science, Yonsei University, Seoul, Republic of Korea.

Received: 7 October 2021 Accepted: 20 January 2022

Published online: 26 January 2022

\section{References}

1. Kottaridis PD, Gale RE, Frew ME, Harrison G, Langabeer SE, Belton AA, et al. The presence of a FLT3 internal tandem duplication in patients with acute myeloid leukemia (AML) adds important prognostic information to cytogenetic risk group and response to the first cycle of chemotherapy: analysis of 854 patients from the United Kingdom Medical Research Council AML 10 and 12 trials. Blood. 2001;98(6):1752-9. https://doi.org/10.1182/ blood.v98.6.1752.

2. Brandts $\mathrm{CH}$, Sargin B, Rode M, Biermann C, Lindtner B, Schwäble J, et al. Constitutive activation of Akt by Flt3 internal tandem duplications is necessary for increased survival, proliferation, and myeloid transformation. Cancer Res. 2005;65(21):9643-50. https://doi.org/10.1158/0008-5472.Can-050422.

3. Levis M, Perl AE. Gilteritinib: potent targeting of FLT3 mutations in AML. Blood Adv. 2020;4(6):1178-91. https://doi.org/10.1182/bloodadvances.201 9000174.

4. Stone RM, Mandrekar SJ, Sanford BL, Laumann K, Geyer S, Bloomfield CD, et al. Midostaurin plus chemotherapy for acute myeloid leukemia with a FLT3 mutation. N Engl J Med. 2017;377(5):454-64. https://doi.org/10.1056/ NEJMoa1614359.

5. Perl AE, Altman JK, Cortes J, Smith C, Litzow M, Baer MR, et al. Selective inhibition of FLT3 by gilteritinib in relapsed or refractory acute myeloid leukaemia: a multicentre, first-in-human, open-label, phase 1-2 study. Lancet Oncol. 2017;18(8):1061-75. https://doi.org/10.1016/s1470-2045(17)30416-3.

6. Döhner H, Estey E, Grimwade D, Amadori S, Appelbaum FR, Büchner T, et al. Diagnosis and management of AML in adults: 2017 ELN recommendations from an international expert panel. Blood. 2017;129(4):424-47. https://doi. org/10.1182/blood-2016-08-733196

7. Murphy KM, Levis M, Hafez MJ, Geiger T, Cooper LC, Smith BD, et al. Detection of FLT3 internal tandem duplication and D835 mutations by a multiplex polymerase chain reaction and capillary electrophoresis assay. J Mol Diagn. 2003;5(2):96-102. https://doi.org/10.1016/s1525-1578(10)60458-8.

8. Thiede C, Steudel C, Mohr B, Schaich M, Schäkel U, Platzbecker U, et al. Analysis of FLT3-activating mutations in 979 patients with acute myelogenous leukemia: association with FAB subtypes and identification of subgroups with poor prognosis. Blood. 2002;99(12):4326-35. https://doi. org/10.1182/blood.v99.12.4326.
9. Pollyea DA, Bixby D, Perl A, Bhatt VR, Altman JK, Appelbaum FR, et al. NCCN guidelines insights: acute myeloid leukemia, version 2.2021. J Natl Compr Cancer Netw. 2021;19(1):16-27. https://doi.org/10.6004/jnccn.2021.0002.

10. Arber DA, Orazi A, Hasserjian R, Thiele J, Borowitz MJ, Le Beau MM, et al. The 2016 revision to the World Health Organization classification of myeloid neoplasms and acute leukemia. Blood. 2016;127(20):2391-405. https://doi. org/10.1182/blood-2016-03-643544.

11. Stirewalt DL, Pogosova-Agadjanyan EL, Tsuchiya K, Joaquin J, Meshinchi S. Copy-neutral loss of heterozygosity is prevalent and a late event in the pathogenesis of FLT3/ITD AML. Blood Cancer J. 2014;4(5):e208. https://doi. org/10.1038/bcj.2014.27

12. Heuser M, Ofran $Y$, Boissel N, Brunet Mauri S, Craddock C, Janssen J, et al. Acute myeloid leukaemia in adult patients: ESMO clinical practice guidelines for diagnosis, treatment and follow-up. Ann Oncol. 2020;31(6):697-712. https://doi.org/10.1016/j.annonc.2020.02.018

13. Sakaguchi M, Nakajima N, Yamaguchi H, Najima Y, Shono K, Marumo A, et al. The sensitivity of the FLT3-ITD detection method is an important consideration when diagnosing acute myeloid leukemia. Leuk Res Rep. 2020;13:100198. https://doi.org/10.1016/j.Irr.2020.100198.

14. DeZern AE, Sung A, Kim S, Smith BD, Karp JE, Gore SD, et al. Role of allogeneic transplantation for FLT3/ITD acute myeloid leukemia: outcomes from 133 consecutive newly diagnosed patients from a single institution. Biol Blood Marrow Transplant. 2011;17(9):1404-9. https://doi.org/10.1016/j. bbmt.2011.02.003.

15. Bornhauser M, IIImer T, Schaich M, Soucek S, Ehninger G, Thiede C, et al. Improved outcome after stem-cell transplantation in FLT3/ITD-positive AML. Blood. 2007;109(5):2264-5; author reply 5. https://doi.org/10.1182/blood-2 006-09-047225.

16. Sengsayadeth SM, Jagasia M, Engelhardt BG, Kassim A, Strickland SA, Goodman S, et al. Allo-SCT for high-risk AML-CR1 in the molecular era: impact of FLT3/ITD outweighs the conventional markers. Bone Marrow Transplant. 2012;47(12):1535-7. https://doi.org/10.1038/bmt.2012.88.

17. Brunet S, Labopin M, Esteve J, Cornelissen J, Socie G, lori AP, et al. Impact of FLT3 internal tandem duplication on the outcome of related and unrelated hematopoietic transplantation for adult acute myeloid leukemia in first remission: a retrospective analysis. J Clin Oncol. 2012:30(7):735-41. https:// doi.org/10.1200/JCO.2011.36.9868

18. Tsai HK, Brackett DG, Szeto D, Frazier R, MacLeay A, Davineni P, et al. Targeted informatics for optimal detection, characterization, and quantification of FLT3 internal tandem duplications across multiple nextgeneration sequencing platforms. J Mol Diagn. 2020;22(9):1162-78. https:// doi.org/10.1016/j.jmoldx.2020.06.006.

19. Wu M, Li C, Zhu X. FLT3 inhibitors in acute myeloid leukemia. J Hematol Oncol. 2018;11(1):133. https://doi.org/10.1186/s13045-018-0675-4.

20. Levine RL, Valk PJM. Next-generation sequencing in the diagnosis and minimal residual disease assessment of acute myeloid leukemia. Haematologica. 2019;104(5):868-71. https://doi.org/10.3324/haematol.2018.2 05955.

21. Spencer DH, Abel HJ, Lockwood CM, Payton JE, Szankasi P, Kelley TW, et al. Detection of FLT3 internal tandem duplication in targeted, short-readlength, next-generation sequencing data. J Mol Diagn. 2013:15(1):81-93. https://doi.org/10.1016/j.jmoldx.2012.08.001

22. Levis MJ, Perl AE, Altman JK, Gocke CD, Bahceci E, Hill J, et al. A nextgeneration sequencing-based assay for minimal residual disease assessment in AML patients with FLT3-ITD mutations. Blood Adv. 2018;2(8): 825-31. https://doi.org/10.1182/bloodadvances.2018015925.

23. Kim B, Kim S, Lee ST, Min YH, Choi JR. FLT3 internal tandem duplication in patients with acute myeloid leukemia is readily detectable in a single nextgeneration sequencing assay using the Pindel algorithm. Ann Lab Med. 2019:39(3):327-9. https://doi.org/10.3343/alm.2019.39.3.327.

24. Kim Y, Lee GD, Park J, Yoon JH, Kim HJ, Min WS, et al. Quantitative fragment analysis of FLT3-ITD efficiently identifying poor prognostic group with high mutant allele burden or long ITD length. Blood Cancer J. 2015;5(8):e336 https://doi.org/10.1038/bcj.2015.61.

25. Grunwald MR, Tseng LH, Lin MT, Pratz KW, Eshleman JR, Levis MJ, et al. Improved FLT3 internal tandem duplication PCR assay predicts outcome after allogeneic transplant for acute myeloid leukemia. Biol Blood Marrow Transplant. 2014;20(12):1989-95. https://doi.org/10.1016/j.bbmt.2 014.08.015.

26. Schranz K, Hubmann M, Harin E, Vosberg S, Herold T, Metzeler KH, et al. Clonal heterogeneity of FLT3-ITD detected by high-throughput amplicon 
sequencing correlates with adverse prognosis in acute myeloid leukemia. Oncotarget. 2018;9(53):30128-45. https://doi.org/10.18632/oncotarget.25729.

27. Yuan D, He X, Han X, Yang C, Liu F, Zhang S, et al. Comprehensive review and evaluation of computational methods for identifying FLT3-internal tandem duplication in acute myeloid leukaemia. Brief Bioinform. 2021;22(5). https://doi.org/10.1093/bib/bbab099.

28. Narzisi G, O'Rawe JA, lossifov I, Fang H, Lee YH, Wang Z, et al. Accurate de novo and transmitted indel detection in exome-capture data using microassembly. Nat Methods. 2014;11(10):1033-6. https://doi.org/10.1038/ nmeth.3069.

\section{Publisher's Note}

Springer Nature remains neutral with regard to jurisdictional claims in published maps and institutional affiliations.

Ready to submit your research? Choose BMC and benefit from:

- fast, convenient online submission

- thorough peer review by experienced researchers in your field

- rapid publication on acceptance

- support for research data, including large and complex data types

- gold Open Access which fosters wider collaboration and increased citations

- maximum visibility for your research: over $100 \mathrm{M}$ website views per year

At $\mathrm{BMC}$, research is always in progress.

Learn more biomedcentral.com/submissions 